Der Physik-Preis 2008 wurde Herrn Thomas Pfohl, Basel und Göttingen, in Würdigung seiner bedeutenden wissenschaftlichen Arbeiten zur Beobachtung und Manipulation von Makromolekülen im Scherfluß auf Mikrometerskale verliehen.

\title{
Analyse, Manipulation und Aggregation von biologischen Makromolekülen im mikrofluidischen Scherfluss
}

\section{Thomas Pfohl}

Die Manipulation, Kontrolle und Untersuchung kleinster Flüssigkeitsmengen im Bereich von Nanound Picolitern haben aufgrund ihres großen Potentials in der Biotechnologie und der Biomedizin weites Interesse erweckt, wodurch in kürzester Zeit nicht nur viele Forschungsdisziplinen verbunden, sondern auch neue Forschungszweige erschlossen wurden. Die Fortschritte auf dem Gebiet der sogenannten Mikro- und Nanofluidik ermöglichen darüber hinaus neuartige Experimente $\mathrm{zu}$ grundlegenden Fragen in der Biophysik, der Physikalischen Chemie und in den Material-

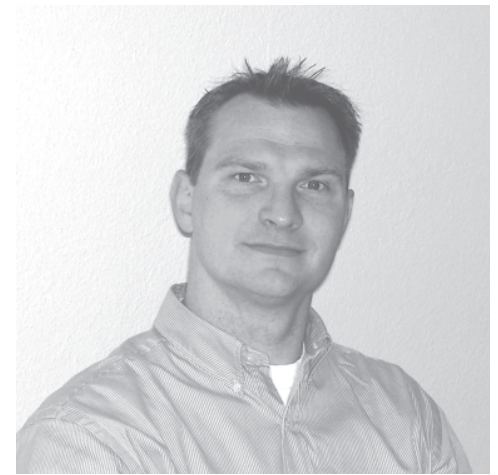

Thomas Pfohl, Professor für Biophysikalische Chemie, Departement Chemie, Universität Basel, und Projektgruppenleiter am Max PlanckInstitut für Dynamik und Selbstorganisation, Göttingen, Träger des Physik-Preises 2008 wissenschaften ${ }^{1}$. So spielen mikrofluidische Anwendungen eine bedeutende Rolle bei der Untersuchung molekularer Wechselwirkungen und chemischer Reaktionen. Sehr kleine Probenvolumina, kurze Reaktions-, Diffusions- und Mischzeiten sowie die Möglichkeit, einzelne (Makro-) Moleküle zu untersuchen, sind die besonders herauszuhebenden Vorteile dieser Methode. Abhängig von den zu untersuchenden Vorgängen bzw. Reaktionen und den experimentellen Techniken (z.B. optische und spektroskopische Mikroskopie, Röntgenstreuung, optische Fallen), verwenden wir unterschiedliche mikrofluidische Methoden, um die Systeme den experimentellen Anforderungen anzupassen. Bei den von uns untersuchten Reaktionssystemen handelt es sich typischerweise um wässrige Lösungen 
von biologischen Makromolekülen wie DNS oder Proteinen, um Zellbestandteile oder sogar ganze Zellen, deren typische Abmessungen mit den Kanaldimensionen des Mikroflusssystems vergleichbar sind. Da die räumliche Beschränkung aufgrund der mikroskopischen Dimensionen großen Einfluss auf die dynamischen Eigenschaften dieser biologischen „weichen“" Objekte besitzt, ist ein detailliertes Verständnis der Strömungsdynamik und des molekularen Transportes auf der Mikro- und der Nanometerskala notwendig. Viele Prozesse, die in lebenden Zellen ablaufen, beispielsweise der intrazelluläre Materialtransport durch das Proteinnetzwerk des Zellskeletts, beinhalten mehr oder weniger stark das Fließen komplexer Flüssigkeiten in Geometrien, deren charakteristische Längenskala der Größe der transportierten Moleküle vergleichbar ist.

Der charakteristische Fluss in mikrofluidischen Experimenten mit typisch erreichten Flussgeschwindigkeiten von wenigen $\mathrm{mm} / \mathrm{s}$ fällt aufgrund der niedrigen Reynoldszahlen in das Regime der laminaren Strömung. Viskose Kräfte dominieren über Trägheitskräfte und lassen keine Turbulenz entstehen. In den langen und schmalen Geometrien der Mikrokanalsysteme bewegt sich die gesamte Flüssigkeit parallel zur örtlichen Orientierung der Kanalwände. Material- und Wärmetransport senkrecht zur Flussrichtung erfolgen im Wesentlichen durch Diffusion und Wärmeleitung ${ }^{1,2}$.

\section{Selbstassemblierungsprozesse im hydrodynamischen Fluss}

Schon mikrofluidische Bauteile mit sehr einfachen Geometrien können für eine große Zahl von unterschiedlichen, sehr aussagekräftigen und grundlegenden Experimenten in der Bio- und der Polymerphysik genutzt werden. Ein ebenso interessantes wie einfaches Bauteil besteht aus zwei gekreuzten Kanälen. Diese Anordnung bietet die Möglichkeit, einen Flüssigkeitsstrahl in der Mikrokanalstruktur hydrodynamisch zu fokussieren, wodurch ein weites Experimentierfeld eröffnet wird ${ }^{3}$. Die Breite des hydrodynamisch fokussierten Strahls lässt sich durch das Verhältnis der Flussraten des Hauptkanals und der beiden Seitenkanäle gezielt einstellen und kann dadurch wesentlich schmaler werden als die tatsächliche Kanalbreite (siehe Abbildung 1). Mit dieser Anordnung können je nach den experimentell eingestellten Parametern sowohl Untersuchungen schneller Reaktionen bei Mischprozessen als auch Reaktionen in Konzentrationsgradienten durchgeführt werden. Die Lösungen beispielsweise mit unterschiedlichen reaktiven Substanzen fließen dabei beim Zusammenfluss laminar nebeneinander. An der Grenzfläche werden diese Substanzen durch molekulare Diffusion ver- 


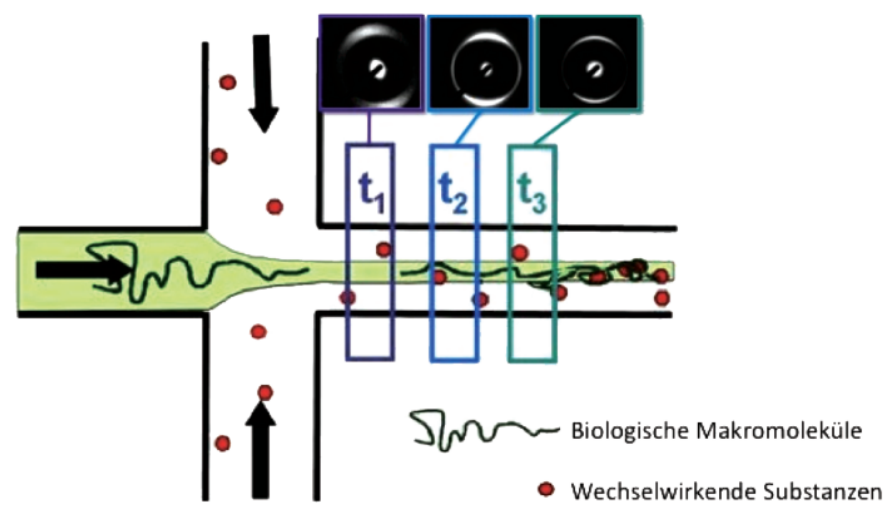

Abbildung 1: Untersuchung von DNS-Kondensation in einem Mikrofluidikbauteil mit hydrodynamischer Fokussierung. Schematischer Aufbau von orts- $(\approx$ zeit-)aufgelösten Röntgendiffraktionsmessungen

mischt und dadurch mögliche Reaktionen zwischen den einzelnen Komponenten gestartet. Die Mischzeiten bzw. Gradienten können durch die Breite des fokussierten Strahls eingestellt werden. Bei einem kontinuierlichen Zufluss der Lösungen mit konstanter Zusammensetzung erreichen die Strömung, der Grad der Durchmischung und der Status der Reaktion einen quasistationären Zustand. Durch Messungen an mehreren, verschieden „flussabwärts“ gelegenen Orten kann die zeitliche Entwicklung oder die Konzentrationsabhängigkeit der Reaktion ermittelt werden ${ }^{4}$.

Mit Hilfe dieser Methodik ist es uns gelungen, die Wechselwirkungen zwischen DNS und Histonen (Proteine, die in Chromosomen u.a. für das dichte Packen der DNS verantwortlich sind) sowie die zwischen DNS und artifiziellen packungsinduzierenden Molekülen, Dendrimeren, die als gleichmäßig geladene, bis zu 10nm große, kationische kugelförmige Moleküle beschrieben werden können, gezielt zu untersuchen ${ }^{5,6}$.

Die Durchführung der Messungen im kontinuierlichen Fluss unter Ausnutzung hydrodynamischer Fokussierungseffekte gewährt einen kontrollierten und zeitaufgelösten Zugang zu den verschiedenen Stadien der Selbstassemblierungsprozesse. Dadurch können insbesondere transiente Zwischenstadien der Reaktion verfolgt und ein quantitatives Verständnis der Packungsmechanismen erzielt werden. Der hohe Grad an Kontrolle über die Größe und die Ladung der Dendrimere ermöglicht es, verschiedene DNS-Packungsszenarien gezielt einzustellen und im Detail zu analysieren. Die hydrodynamische Fokussierung führt darüber hinaus zu einer zusätz- 
lichen Orientierung und Streckung der assemblierten Strukturen, die eine Charakterisierung und Analyse mit Messmethoden wie der Fluoreszenz-, der Polarisations-, der Ramanmikroskopie sowie der Röntgenkleinwinkelstreuung vereinfachen oder teilweise erst ermöglichen.

In weiteren Experimenten konnten wir durch die Generierung eines stabilen $\mathrm{pH}-$ Gradienten die dynamische Entwicklung der hierarchischen Organisation von Kollagenfibrillen auf der Nanometerskala analysieren ${ }^{7}$. Diese weitgehende Flexibilität an Experimenten zeigt das große Potential, das mikrofluidische Methoden zur Analyse der Dynamik von Selbstassemblierungs- und von Selbstorganisationsprozessen besitzen.

\section{Einzelne Polymerfilamente im mikroskopischen Scherfluss}

Aktin, ein Protein des intrazellulären Fasernetzwerks, das aufgrund seiner Steifheit zu den semiflexiblen Polymeren gezählt wird, stellt ein vorzügliches Modellfilament für Untersuchungen zum Einfluss geometrischer Einschränkung und zu mikroskopischen Strömungen dar ${ }^{8}$. Einzelne Aktinfilamente $(\sim 8 \mathrm{~nm}$ Durchmesser mit einer Länge von mehreren $\mu \mathrm{m})$ lassen sich in zellenfreien Medien polymerisieren und können durch Fluoreszenzmarkierung direkt mit Hilfe der Fluoreszenzmikroskopie beobachtet werden. Da die Maschenweite von Netzwerken des Zellenskeletts wie auch der zellenumgebenden Proteinmatrix im Bereich weniger Mikrometer liegt, ist die Mikrofluidik besonders geeignet, um einerseits das prinzipielle Verhalten von Einzelmolekülen, die von einem Geflecht aus Polymeren umgeben sind, und andererseits das Zusammenspiel und die Organisation der Netzwerkbestandteile zu charakterisieren ${ }^{9}$. Eine Analyse einzelner fluktuierender Filamente, deren Bewegung durch einschränkende Kanäle und Strömungsfelder beeinträchtigt ist, ermöglicht eine umfassende Beschreibung der mechanischen Systemeigenschaften ${ }^{8,10}$.

Zur fluoreszenzmikroskopischen Abbildung von strömenden Filamenten in Mikrokanälen muss ein Aufbau mit stroboskopischer Laseranregung gewählt werden, um sehr kurze Belichtungsdauern zu erreichen, damit Filamentkonturen ohne „Verschmierungen“ visualisiert werden können ${ }^{11}$. Im Vergleich zu den Untersuchungen der Filamente in einschränkenden Geometrien (Mikrokanal ohne Strömung) sorgt eine angelegte Strömung entweder für eine zusätzliche Streckung oder für eine Verbiegung der Filamente (siehe Abb. 2). Die Verstreckung oder Verbiegung der Filamente kann direkt auf die Strömungsfelder und den Scherfluss in den Mikrokanälen zurückgeführt werden. Mit zunehmender Geschwindigkeit nimmt 
a)

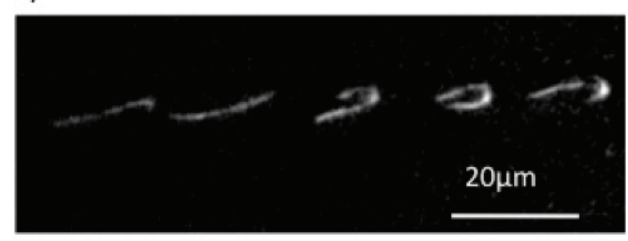

b)

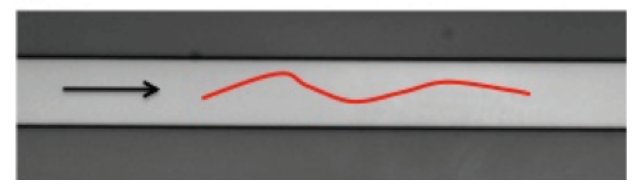

Abbildung 2: a) Stroboskopische Aufnahme von strömenden Aktinfilamenten in Mikrokanälen; b) Skizze des Versuchaufbaus

die Verstreckung der gestreckten Filamente zu, während der Biegeradius der gebogenen Filamente abnimmt. Zusätzlich können „taumelnde“ Filamente beobachtet werden, bei denen das eine Ende eines Filaments das andere Ende überholt. Diese unterschiedlichen Konformationen und Orientierungen haben direkten Einfluss auf die Häufigkeit, Filamente an bestimmten Kanalpositionen entlang einem Querschnitt zu finden. Die Wahrscheinlichkeitsverteilungen der Schwerpunkte zeigen, dass die Filamente nicht gleichmäßig über die ganze Kanalbreite verteilt sind. In der Nähe der Kanalwände existieren deutliche Verarmungszonen. Bei hohen Flussgeschwindigkeiten bildet sich zusätzlich eine starke Verarmungszone in der Kanalmitte aus, und die meisten Filamente werden bei etwa Einviertel bzw. Dreiviertel der Kanalbreite gefunden. Diese stromlinienübergreifende Migration der Filamente lässt sich auf eine scherratenabhängige Segmentmobilität der Filamente in der Mikrokanalströmung zurückführen. Diese Experimente geben Einblicke nicht nur in die Physik der Polymere unter räumlicher Einschränkung und Scherfluss, die für Anwendungen in der Mikrofluidik sowie der Polymerherstellung und -bearbeitung eine wichtige Rolle spielen, sondern auch in biophysikalische Eigenschaften von Blut und dessen Bestandteilen beim Strömen durch Blutgefässe. So scheinen sich Trypanosomen, einzellige Erreger der Schlafkrankheit, durch hydrodynamische Wechselwirkungen im strömenden Blut gegen das Immunsystem des infizierten Säugertiers zu verteidigen ${ }^{12}$, indem sie sich höchstwahrscheinlich selbst im Blutstrom nahe an den Blutgefässwänden positionieren, um in einem weiteren Schritt der Infektion die Blut-Hirnschranke zu überwinden. 
All diese Experimente führen nicht nur zu einer Erweiterung des fundamentalen Verständnisses der untersuchten biophysikalischen Systeme, sondern zeigen auch das Potential mikrofluidischer Techniken auf, umfassende Bereiche der Lebenswissenschaften weiter zu entwickeln.

\section{Literatur}

1. T. Pfohl, F. Mugele, R. Seemann, S. Herminghaus, ChemPhysChem 4, 1291 (2003).

2. T.M. Squires and S.R. Quake, Review of Modern Physics 77, 977 (2005).

3. J.B. Knight, A. Vishwanath, J.P. Brody, R.H. Austin, Phys. Rev. Lett. 1998, 80, 3863.

4. H.M. Evans, R. Dootz, S. Köster, B. Struth, T. Pfohl, Bulletin of the Polish Academy of Science - Technical Sciences 55, 217 (2007).

5. R. Dootz, A. Otten, S. Köster, B. Struth, T. Pfohl, „Evolution of DNA compaction in microchannels", Journal of Physics: Condensed Matter 18, S639-S652 (2006).

6. T. Pfohl, A. Otten, S. Köster, R. Dootz, B. Struth, H.M. Evans, Biomacromolecules 8, 2167 (2007).

7. S. Köster, H.M. Evans, J.Y. Wong, T. Pfohl, Biomacromolecules 9, 199 (2008).

8. S. Köster, J. Kierfeld, T. Pfohl, European Physical Journal E 25, 439 (2008).

9. S. Köster and T. Pfohl, Cell Motility and the Cytoskeleton 66, 771 (2009).

10. S. Köster, D. Steinhauser, T. Pfohl, Journal of Physics: Condensed Matter 17, S4091 (2005).

11. D. Steinhauser, Dissertation, Georg-August-Universität, Göttingen, http://webdoc.sub.gwdg.de/diss/2008/steinhauser-/steinhauser.pdf (2008).

12. M. Engstler, T. Pfohl, S. Herminghaus, M. Boshart, G. Wiegertjes, Niko Heddergott, P. Overath, Cell 131, 505 (2007). 